\title{
SYNCHROTRON AND LABORATORY STUDIES UTILIZING A NEW \\ POWDER DIFFRACTION TECHNIQUE*
}

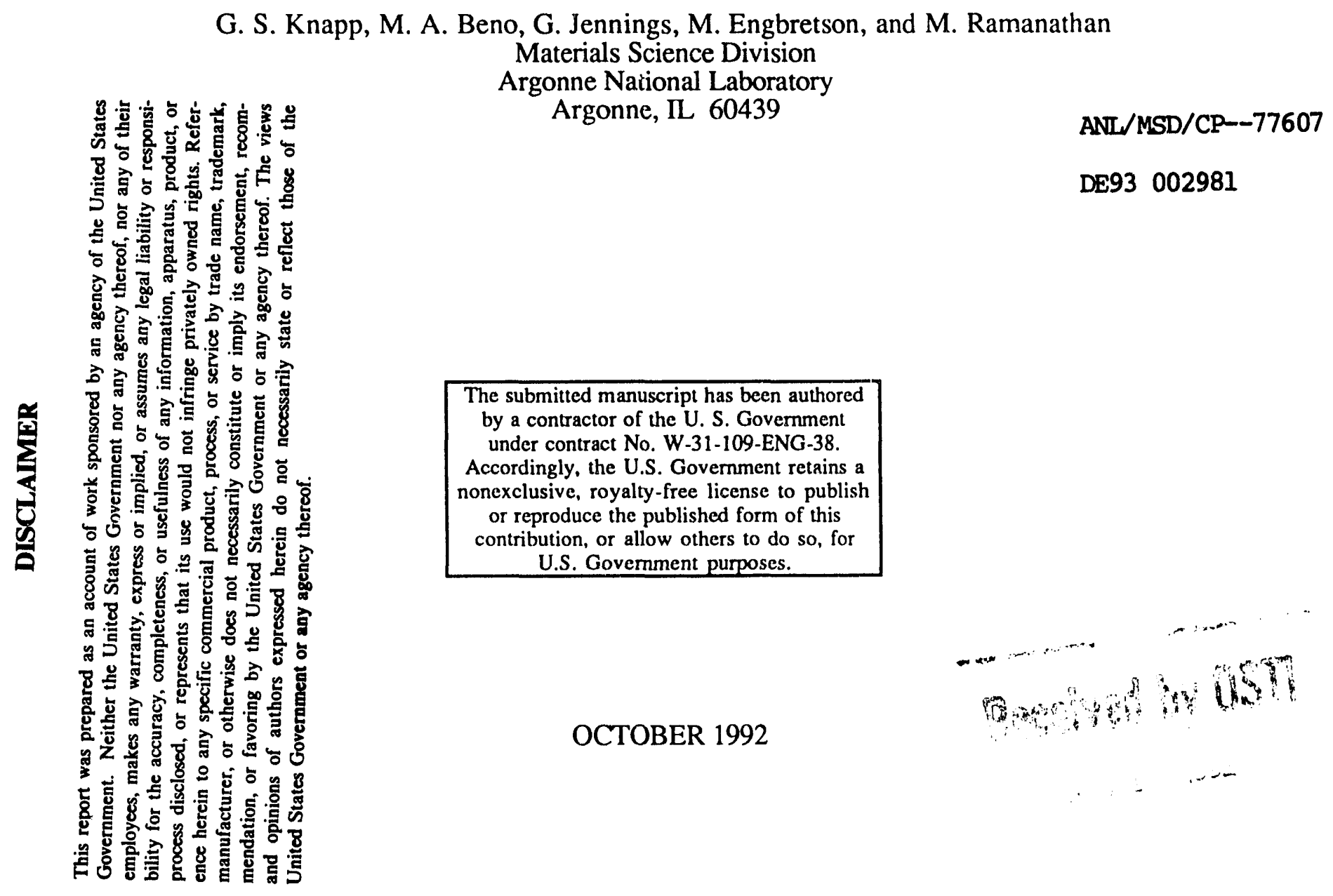

Submitted to 41st Annual Denver X-Ray Conference on Applications of X-Ray Analysis, Colorado Springs, CO, August 3-7, 1992.

\footnotetext{
* Work Supported by the U.S. Department of Energy, BES-Materials Sciences, under Contract \#W-31-109-ENG-38. 


\title{
SYNCHROTRON AND LABORATORY STUDIES UTILIZING A NEW POWDER DIFFRACTION TECHNIQUE
}

\author{
G. S. Knapp, M. A. Beno, G. Jennings, M. Engbretson and \\ M. Ramanathan \\ Argonne National Laboratory, Argonne IL 60439.
}

\begin{abstract}
We have developed a new type of powder diffractometer that is much more efficient than existing methods. The diffractometer has the potential of both high count rates and very high resolution when used at a synchrotron source. The laboratory based instrument has an order of magnitude improvement in count rate over existing methods. The method uses a focusing diffracted beam monochromator in combination with a multichannel detector. The incident $x$-rays fall on a flat plate or capillary sample and are intercepted by a bent focusing monochromator which has the focus of the bend at the sample surface. The powder diffraction lines emerging from the bent crystal monochromator are detected by a linear or 2dimensional detector. This allows us to eliminate the background from fluorescence or other scattering and to take data over a range of $3^{\circ}$ to $4^{\circ}$ instead of one angle at a time thereby providing a large improvement over conventional diffractometers.
\end{abstract}

\section{INTRODUCTION}

Powder Diffraction is one of the most widely used analytical techniques. The technique is useful not only to study crystalline materials but also to study amorphous materials. When used with a synchrotron source the phenomena of anomalous scattering can be exploited which results in a great deal more information than can be obtained from single wavelength studies alone. Unfortunately, the most widely used diffractometer type, the Bragg Brentano ${ }^{1,2}$ diffractometer gives quite low count rates, so we have developed a method that is much more efficient than existing methods. This increase in efficiency is very important for time resolved studies as well as studies of materials that contain trace phases. The method combines the advantages of two of the most important powder diffraction techniques, the Bragg Brentano method and the multichannel detector method. The best 
Bragg Brentano diffractometers use a diffracted beam monochromator to eliminate air scatter and fluorescence, but these types of instruments use a single channel detector so the data is taken one angle at a time and many angles must be scanned for a complete set of data. The best multichannel detectors 3,4 are curved and they cover up to $120^{\circ}$ in 2-theta. Unfortunately, since the multichannel approach does not use a diffracted beam monochromator the backgrounds can be high, particularly if the material being studied contains elements that fluoresce. This greatly limits the usefulness of this method. Below we will discuss our new method 5 which employs somewhat different optics when used with a synchrotron or a laboratory source. We will discuss each system separately and we will compare our new method to existing techniques.

\section{LABORATORY SOURCE}

A Bragg-Brentano type diffractometer is shown schematically in figure 1A. The diffracted beam monochromator eliminates most of the background caused by sample fluorescence and air scatter. This type of instrument possesses high sensitivity, trace phases as low in concentration as $0.1 \%$ have been determined with this method. The alternative technique utilizes a position sensitive detector that covers a large solid angle. As shown in figure $1 \mathrm{~B}$, position sensitive detectors observe a large portion of the diffraction pattern simultaneously and therefore can achieve rapid collections of $x$-ray powder patterns. However, these detectors do not have energy resolution and therefore can suffer from high backgrounds particularly for materials containing elements which fluoresce. This greatly limits the utility of the position sensitive detector method. In figure $1 \mathrm{C}$ we show the new technique as applied to a laboratory source. In this case we take advantage of sample parafocusing 6 as much as possible in order to maximize flux. Consider figure $1 \mathrm{C}$, which shows two diffracted lines that occur within the acceptance window of the crystal. Both lines are reflected and detected at different positions on the multichannel detector. Currently, angular acceptances of more than $4^{\circ}$ in 2-theta have been achieved using a Johansson 7 cut LiF crystal. A subtle point is that the focusing crystal must have sufficient mosaic spread to take advantage of sample parafocusing. The crystal will accept the convergent beams only up to its mosaic spread width. In addition, in order to be efficient the monochromator must have a uniform mosaic spread or the lines will be poorly diffracted over some parts of the monochromator crystal. The best monochromators we now have possess a diffraction efficiency of only a few percent. We are working on improvements in crystal fabrication which will greatly enhance this performance.

To illustrate the method figure 2 shows the data taken with this method as compared to the results obtained with a standard Bragg-Brentano diffractometer. The diffraction pattern of fluorapatite, $\mathrm{Ca}_{5} \mathrm{~F}\left(\mathrm{PO}_{4}\right)_{3}$, was taken using this technique and then run using a Bragg-Brentano diffractometer ${ }^{8}$ (fig 1A) with the same current and voltage settings ( 30 ma and $45 \mathrm{kV}$ respectively). The data was taken to have the same number of steps and for 
the same number of paak counts. The results are shown in figure 2 for a group of low angle lines. The data took 11 minutes to run with the new

A.

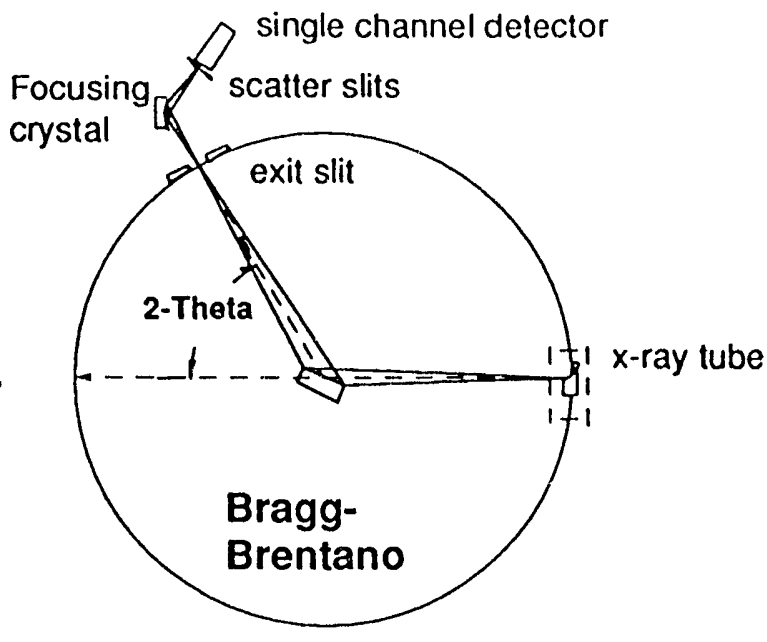

A) A standard BraggBrentano diffractometer employing a focusing analyzer crystal. The sample parafocuses the beam back on the exit slit.

120 degree detector

B.

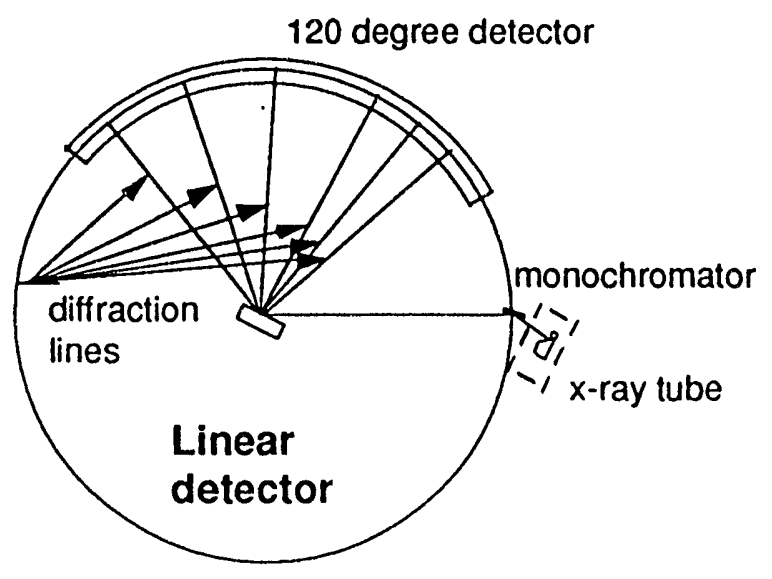

B) A diffractometer employing a curved position sensitive detector.

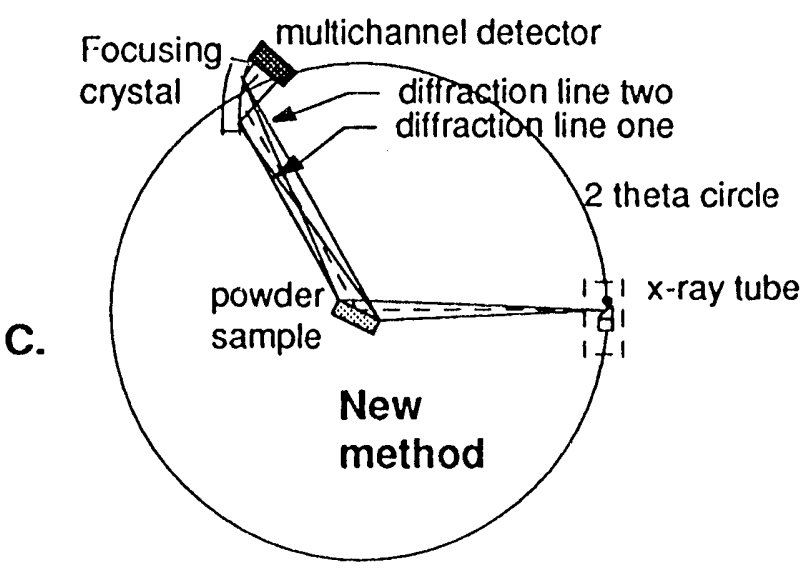

C) A laboratory diffractometer employing a focusing crystal and multichannel detector to observe multiple diffraction lines simultaneously. The concept of parafocusing for this instrument is illustrated in the figure by two different diffraction lines being simultaneously detected.

Fig. 1 Schematic representations of powder diffractometers. 


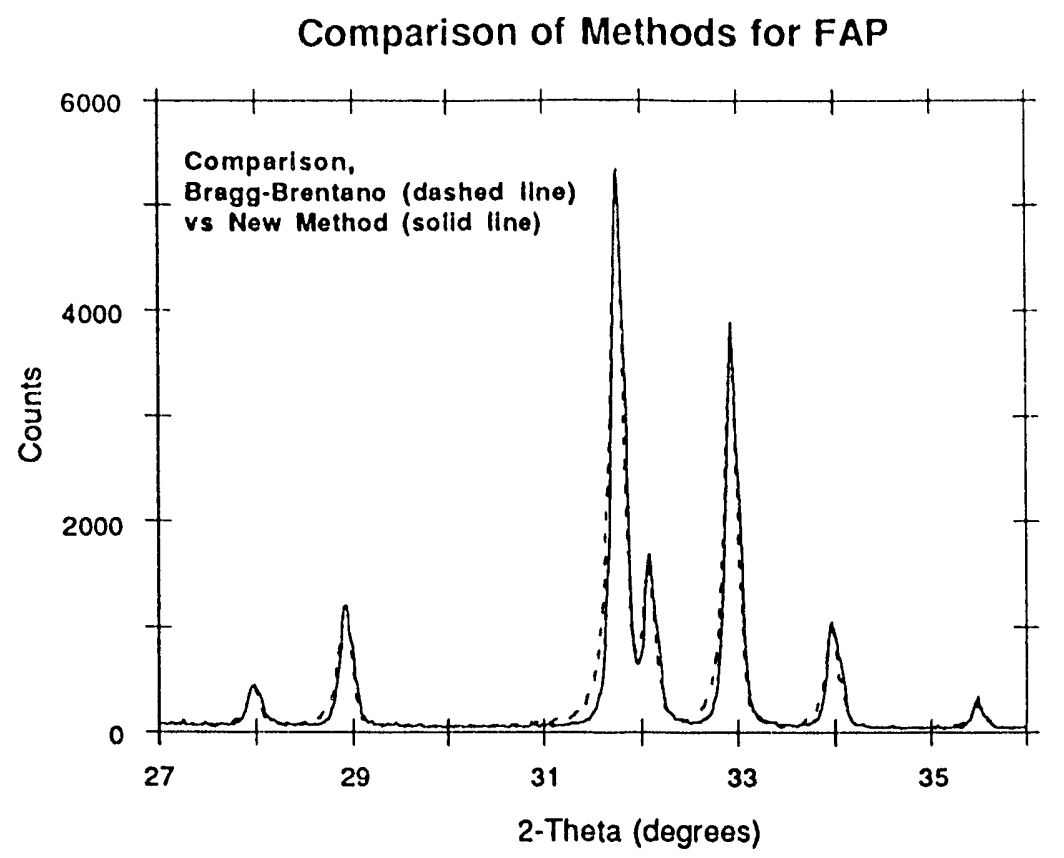

Fig 2. The diffraction patterns of FAP run by the Bragg-Brentano method (dashed line) and the new method (solid line) with the same signal to noise.

method as compared to 55 minutes using the conventional technique so that the data collection speed is 5 times faster and as seen from figure 2 the resolution and the peak shapes are better. The method is not only fast but also achieves low backgrounds. With this method the peak to background ratio for the 111 peak of $\mathrm{CeO}_{2}$ is better than 1000 to 1 . Figure 3 shows the diffraction pattern for $\mathrm{Fe}_{2} \mathrm{O}_{3}$, a material that exhibits extreme fluorescence with $\mathrm{Cu} \mathrm{Ka}$ radiation. Clearly, the method is very efficient in eliminating background.

Using our current monochromator, the increase in data collection speed is a factor of five. This is a major improvement, equivalent to the improvement one gets by changing from a sealed tube to a rotating anode source and it is much cheaper to implement. However, if you consider that when we ran the standard method we took data in $0.02^{\circ}$ intervals and our method took data in $3^{\circ}$ intervals we should expect a factor of 150 improvement. The reason our results are not better is the problems with the mosaic spread of the LiF we are currently using. We expect great improvements both in count rate and in resolution when better crystals are fabricated.

One of the most interesting illustrations of how the technique works is a time resolved study of the high temperature superconductor, GdBaSrCu3O7-x. Work by Veal et. al. ${ }^{9}$ has shown that this type of material will show dramatic changes in superconducting transition temperature when 


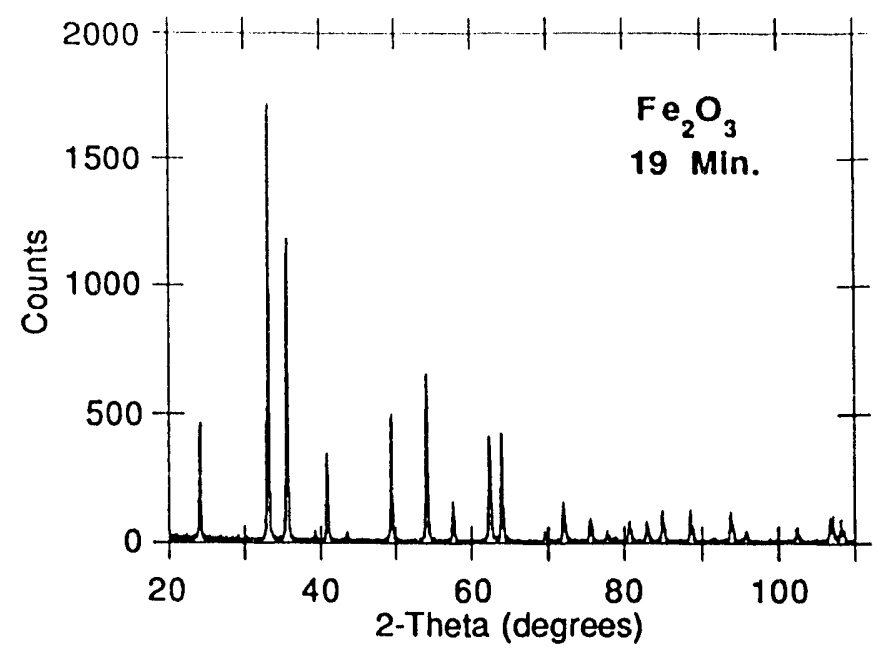

Fig. 3 A diffraction pattern of $\mathrm{Fe}_{2} \mathrm{O}_{3}$ run in 19 minutes at $45 \mathrm{kV}$ and 150 ma. Note the low background.

annealed at very low temperatures. We have studied the time dependence of the structural parameters of this material when we anneal it at $205^{\circ} \mathrm{C}$. In the early stages of annealing data was taken over a 2-theta range of $20^{\circ}$ to $105^{\circ}$ every 33 minutes. In this case we used a rotating anode source operating at 45 $\mathrm{kV}$ and $150 \mathrm{~mA}$. Figure 4 shows the time dependence of the unit cell

\section{GdBaSrCu307}

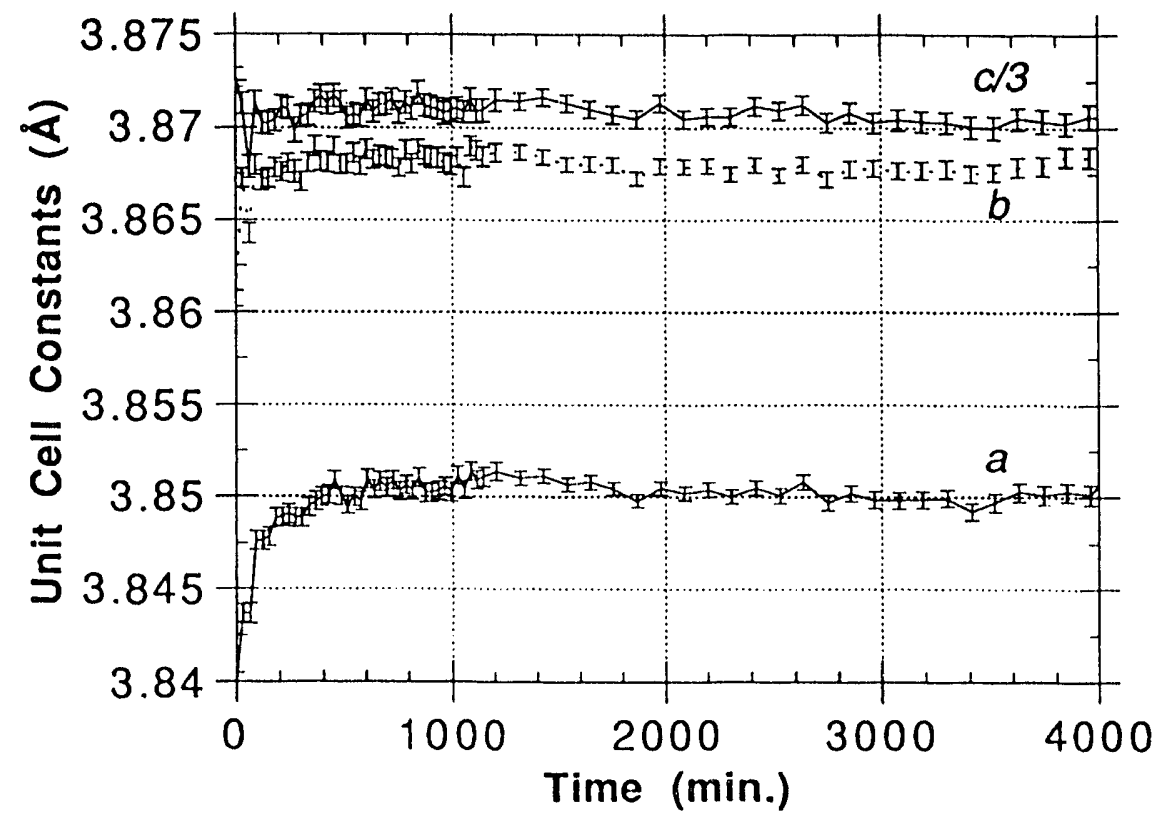

Fig. 4 Time dependent structural changes in $\mathrm{GdBaSrCu}_{3} \mathrm{O} 7-\mathrm{x}$ when the sample is annealed at $205^{\circ} \mathrm{C}$. 
parameters. Note that most of the change in these parameters take place in the first 2 hours of the annealing. Rietveld refinements ${ }^{10}$ of these data give indications of how the atomic positions change with time during the annealing. However, more sensitivity is needed before conclusions about the origins of $T_{C}$ changes can be based on the subtle trends observed. Fortunately there is great potential to improve the technique since the LiF analyzer crystals we are currently using have a very non-uniform reflectivity and poor brightness. We are currently developing a new type of analyzer crystal which should be significantly better in brightness, uniformity and angular range.

\section{SYNCHROTRON SOURCE}

In order to achieve higher flux, improved resolution and the ability to do anomalous scattering we need to develop a synchrotron based system. In figure $5 \mathrm{~A}$ we show the optics of the best single channel synchrotron based system. The method uses an unfocused monochromatic beam and a flat diffracted beam monochromator. The method is somewhat limited in flux and is not very good for small samples but has extremely high resolution. Figure 5B shows the new method on an unfocused beamline. In this case the incoming beam is quite parallel, therefore it is not possible to use parafocusing as in figure $1 \mathrm{C}$ and a focusing diffracted beam monochromator with a large mosaic spread (such as the LiF monochromator used for a lab source) does not work very well since the parallel beams are not efficiently diffracted by the mosaic crystal. In addition, the use of mosaic crystals degrades resolution. Therefore, we have attempted to use perfect crystal Si or Ge focusing monochromators. For an unfocused synchrotron beam (as is depicted in figure $5 B$ ) perfect crystal focusing optics have a field of view that is effectively limited by the Darwin width of the crystal so that only a small portion of the incoming $x$-rays are detected. Nevertheless the method works, achieves a reasonable count rate and very high resolution. In figure 6 we show the results of a run of FAP run on beamline $X 6 \mathrm{~B}$ at the National Synchrotron Light Source (NSLS). This data took 33 minutes to acquire and the resolution was limited only by the detector resolution. Comparison of the count rate we obtained to that reported by Cox ${ }^{11}$ show that the methods shown schematically in figures $5 \mathrm{~A}$ and $5 \mathrm{~B}$ produce roughly comparable count rates. Figure $5 \mathrm{C}$ illustrates how the method can be improved. It is necessary to focus the beam from the synchrotron in order to bring the flux within the field of view of the diffracted beam focusing crystal. It seems possible to bring most of the flux to within the field of view and with focusing in both the vertical and horizontal directions the count rate should improve by about a factor of 150 with only a slight loss of resolution. We will be installing a focusing mirror at beamline $X 6 \mathrm{~B}$ in the next few months and should then be able to test this concept. With this improvement in count rate, we will saturate our current detector. We are developing a CCD detector which will be able to handle the much larger count rates. 

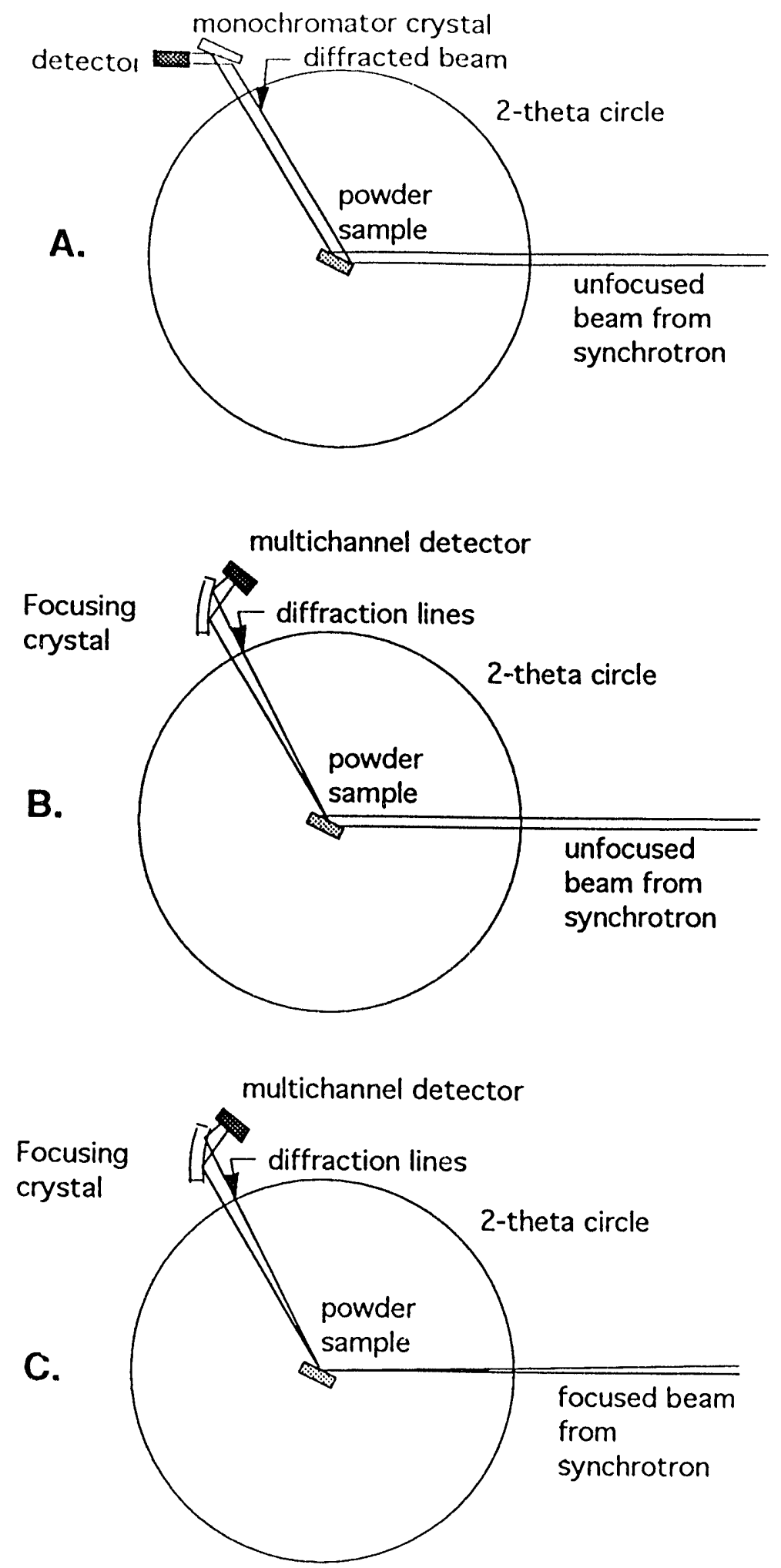

Fig. 5 Schematic drawings of synchrotron powder diffraction techniques; A. unfocused $\mathrm{x}$-ray beam and flat analyzer, Focusing analyzer with unfocused beam, C. Focused synchrotron beam and focusing analyzer crystal, note the match between the field-of-view and illuminated area. 


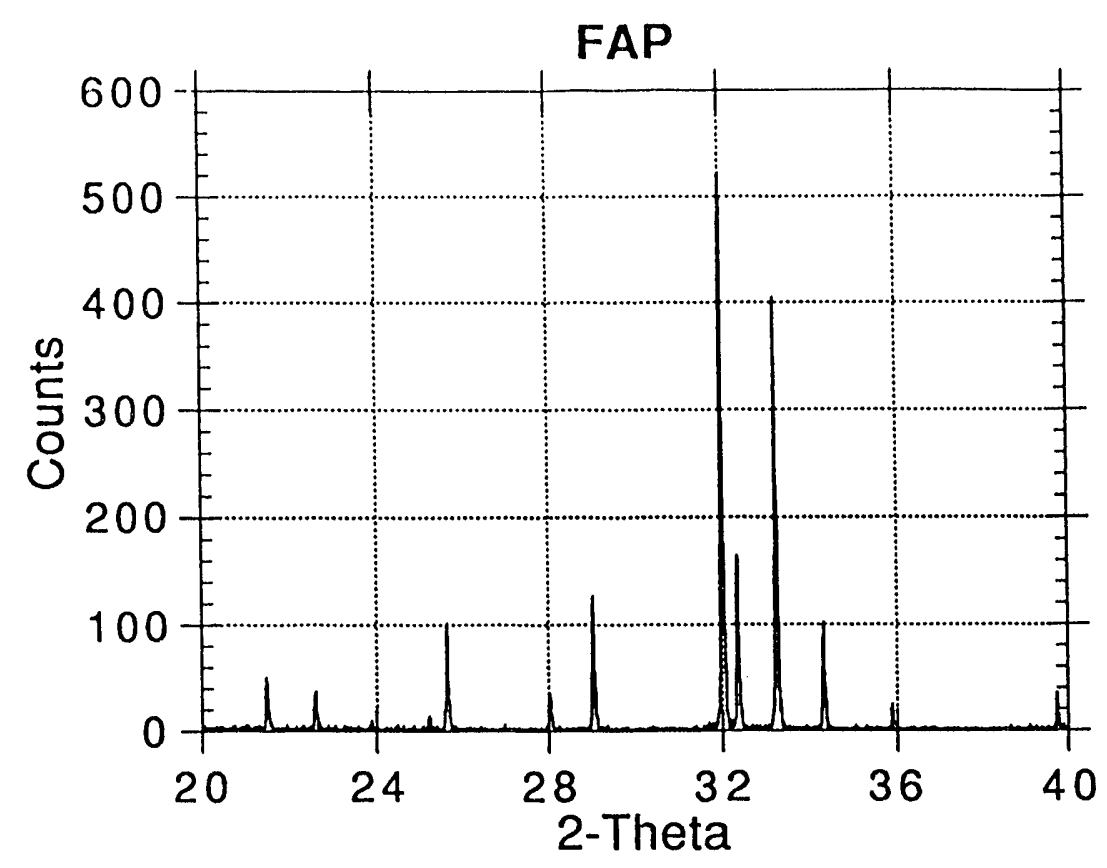

Fig. 6 Synchrotron powder diffraction data for $\mathrm{Ca} 5 \mathrm{~F}\left(\mathrm{PO}_{4}\right)_{3}$ taken with a focusing Si 220 analyzer crystal. The data collection time was $33 \mathrm{~min}$ at $120 \mathrm{ma}$ beam current.

In what times should we be able to resolve time dependent structure changes with this method? Currently, using a mosaic crystal monochromator, we are able to get adequate signal to noise on a set of high angle lines in the order of 10 seconds. With improved optics at NSLS we should be able to reduce this time to approximately 0.1 second. When the Advanced Photon Source (APS) becomes available, the system on a bending magnet beamline should be a factor of 10 better yet, and if the system was mounted on an undulator line another factor of 50 is expected. This means that time resolved studies can be carried out in microseconds. In addition the higher critical energy of APS means that many more edges are available so anomalous scattering studies can be carried out quickly and easily. The higher energies also mean that much larger q ranges can be explored so that radial distribution studies of amorphous and crystalline materials ${ }^{12}$ become feasible. 
ACKNOWLEDGMENTS

Work at Argonne National laboratory is sponsored by the US Department of Energy, Office of Basic Energy Sciences, Division of Materials Sciences, under contract W-31-109-ENG-38. This work was carried out in part at the X6B beamline, National Synchrotron Light Source, Brookhaven National Laboratory, which is supported by the Divisions of Materials Sciences and Chemical Sciences of DOE.

\section{REFERENCES}

1. W.H. Bragg, Proc. Phys. Soc. Lond., 52, 222 (1921).

2. J. Brentano, Arch. Sci. Phys. Nat., 44, 66 (1917).

3. J.Ballon, V. Comparat, and J. Pouxe Nuclear Instruments and Methods, 217, 213 (1983).

4. An example of this type of detector is the Inel CPS 120 System, detector is marketed by Intel Buc France.

5. G. S. Knapp and M. A. Beno, U. S. Patent Application Serial No. 07/811,060, Filed December 19, 1991.

6. For an explanation of parafocusing see, for example H. P. Klug and L. E. Alexander , "X-ray Diffraction Procedures",John Wiley and Sons, Inc. New York, London, 211-218, (1954).

7. T. Johansson, Naturwissenschaften 20, 758 (1932).

8. We use a Bragg-Brentano type diffractometer marketed by Rigaku USA, Inc. Danvers MA, USA.

9. B. W. Veal, A. P. Paulikas, H. You, H. Shi, Y. Fang, and J. W. Downey, Phys. Rev., B42, 6305 (1990).

10. GSAS, General Structure Analysis System, A. C. Larson and R. B. Von Dreele, LAUR 86-748, (1990).

11. D. E. Cox, in "Handbook on Synchrotron Radation" G.S. Brown and D. E. Moncton Editors, North-Holland, Elsevier Science Publishing, New York, 187-188, (1991).

12. B. H. Toby and T. Egami, Acta Cryst., A48, 336 (1992). 

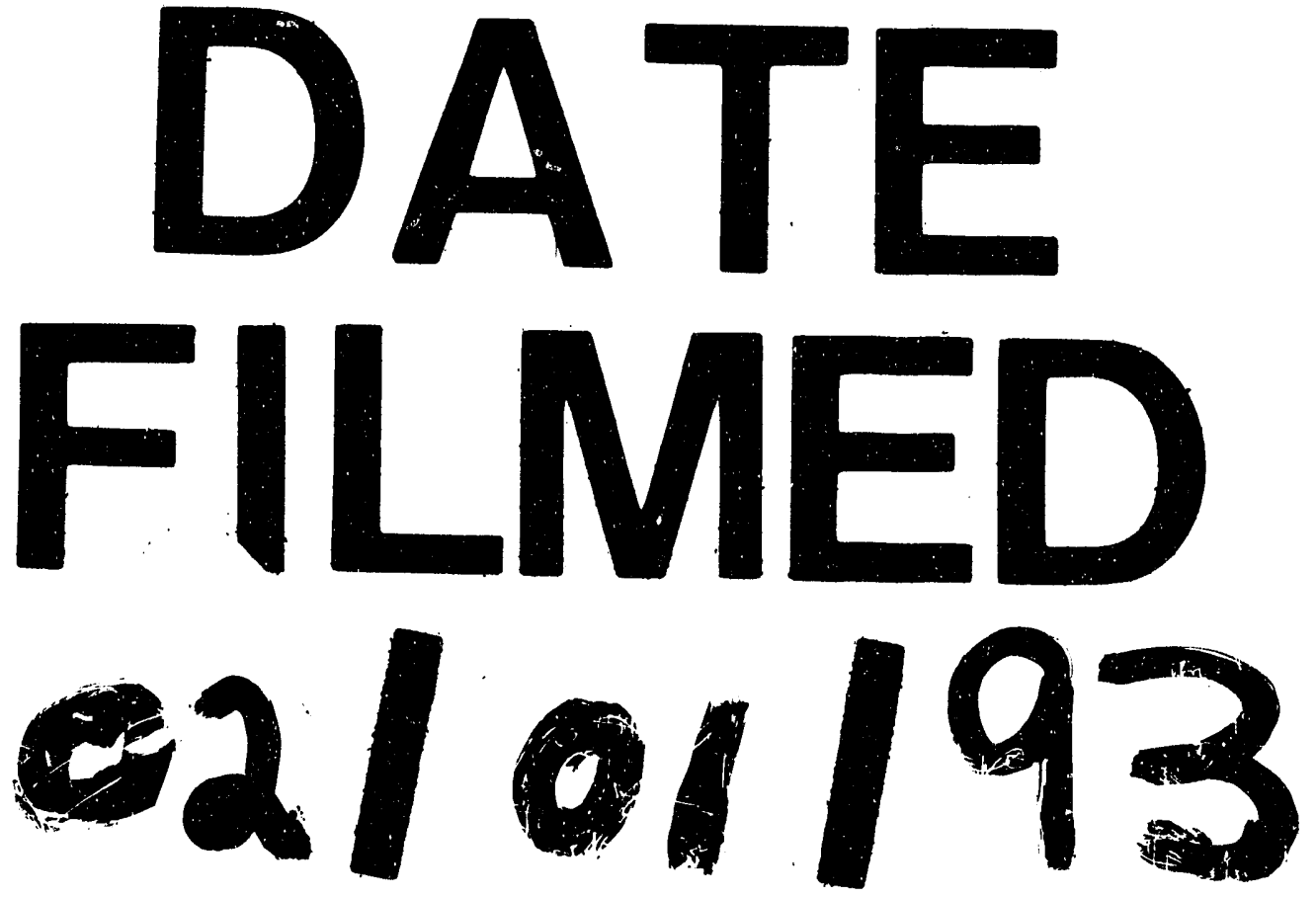
